\title{
SYMPLECTIC MODULAR COMPLEMENTS
}

BY

\section{IRVING REINER}

Introduction. Let $\Omega_{n}$ denote the group of $n \times n$ integral matrices of determinant \pm 1 (the unimodular group), and let $I^{(n)}$ be the identity matrix in $\Omega_{n}$. We use $X^{\prime}$ to represent the transpose of $X$, and $X \dot{+} Y$ for the direct sum of $X$ and $Y$.

The symplectic modular group $\left.{ }^{1}\right) \Gamma_{2 n}$ is the group of $2 n \times 2 n$ integral matrices $\mathfrak{M}$ such that

$$
\mathfrak{M}\left(\begin{array}{cc}
0 & I^{(n)} \\
-I^{(n)} & 0
\end{array}\right) \mathfrak{M}^{\prime}=\left(\begin{array}{cc}
0 & I^{(n)} \\
-I^{(n)} & 0
\end{array}\right) .
$$

A primitive integral $(j+k) \times 2 n$ matrix

$$
\left(\begin{array}{ll}
A_{1}^{(j, n)} & B_{1}^{(j, n)} \\
C_{1}^{(k, n)} & D_{1}^{(k, n)}
\end{array}\right)
$$

in which

$$
A_{1} B_{1}^{\prime} \text { and } C_{1} D_{1}^{\prime} \text { are symmetric }
$$

and

$$
A_{1} D_{1}^{\prime}-B_{1} C_{1}^{\prime}=\left(\begin{array}{ll}
I^{(j)} & 0)
\end{array} \text { or }\left(\begin{array}{c}
I^{(k)} \\
0
\end{array}\right)\right.
$$

(depending on whether $j \leqq k$ or $j \geqq k$ ) will be called a normal $(j, k)$ array. A normal $(j, 0)$ array will be called a normal pair. Then $\Gamma_{2 n}$ is known to consist of all normal $(n, n)$ arrays.

In this paper we shall consider the problem of completing a normal $(j, k)$ array to an element of $\Gamma_{2 n}$ by placing $(n-j)$ rows after the first $j$ rows, and $(n-k)$ rows after the last $k$ rows. Since a sub-array of a normal array is normal, it is clear that an array cannot be completed unless it is normal. It will be shown that every normal array may be so completed, and a parametrization of the general completion will be obtained. These results will generalize those due to $\mathrm{C}$. L. Siegel ( $\left.{ }^{2}\right)$ for the special case $j=n, k=0$, but the proofs given here will not depend on his results.

Presented to the Society, September 3, 1953; received by the editors August 4, 1953.

(1) It is sometimes more convenient to define the symplectic modular group as the factor group of $\Gamma_{2 n}$ over its centrum. See C. L. Siegel, Math. Ann. vol. 116 (1939) pp. 617-657; L. K. Hua, Trans. Amer. Math. Soc. vol. 57 (1945) pp. 441-490; L. K. Hua and I. Reiner, Trans. Amer. Math. Soc. vol. 65 (1949) pp. 415-426.

(2) C. L. Siegel, Ann. of Math. vol. 36 (1935) p. 592. 
1. Let $\mathfrak{X}_{1}, \mathfrak{X}_{2}$ be arrays of the type given in (1); we write $\mathfrak{X}_{1} \sim \mathfrak{X}_{2}$ if there exists $\mathfrak{V} \in \Gamma_{2 n}$ such that $\mathfrak{X}_{1}=\mathfrak{X}_{2} \mathfrak{Z}$. This relationship is an equivalence relationship, and we have:

LEMma 1. Let $\mathfrak{X}_{1}$ be a normal array and $\mathfrak{X}_{2} \sim \mathfrak{X}_{1}$. Then $\mathfrak{X}_{2}$ is also a normal array, and $\mathfrak{X}_{2}$ can be completed if and only if $\mathfrak{X}_{1}$ can be completed.

Proof. Clear.

Before proceeding to the next lemma, it will be convenient to single out certain elements of $\Gamma_{2 n}$ which play the same role in $\Gamma_{2 n}$ as do the elementary transformations in $\Omega_{n}$. Specifically, we define three types of elements of $\Gamma_{2 n}$ :

(I) Translations

$$
\mathfrak{T}_{S}=\left(\begin{array}{ll}
I & S \\
0 & I
\end{array}\right)
$$

$S$ symmetric.

(II) Rotations

$$
\Re_{U}=\left(\begin{array}{cc}
U & 0 \\
0 & U^{\prime-1}
\end{array}\right), \quad U \in \Omega_{n}
$$

(III) Semi-involutions

$$
\mathfrak{S}_{J}=\left(\begin{array}{cc}
J & I-J \\
J-I & J
\end{array}\right), \quad J \text { diagonal with elements 0's and 1's. }
$$

LEMmA 2. If G.C.D. $\left(a_{1}, \cdots, a_{n}, b_{1}, \cdots, b_{n}\right)=1$, then

$$
\left(a_{1}, \cdots, a_{n}, b_{1}, \cdots, b_{n}\right) \sim(1,0, \cdots, 0,0, \cdots, 0) .
$$

Proof. We first observe that

$$
\left(a_{1}, \cdots, a_{n}, b_{1}, \cdots, b_{n}\right) \Re_{U}=\left(\left(a_{1}, \cdots, a_{n}\right) U,\left(b_{1}, \cdots, b_{n}\right) U^{\prime-1}\right)
$$

for $U \in \Omega_{n}$. If we set $a_{0}=$ G.C.D. $\left(a_{1}, \cdots, a_{n}\right)$, by proper choice of $U$ we obtain

$$
\left(a_{1}, \cdots, a_{n}, b_{1}, \cdots, b_{n}\right) \sim\left(a_{0}, 0, \cdots, 0, b_{1}, \cdots, b_{n}\right)
$$

for some integers $b_{1}, \cdots, b_{n}$. Let $b_{0}=$ G.C.D. $\left(b_{2}, \cdots, b_{n}\right)$; then the above reasoning with $U=1+U_{1}, U_{1} \in \Omega_{n-1}$, shows that

$$
\left(a_{1}, \cdots, a_{n}, b_{1}, \cdots, b_{n}\right) \sim\left(a_{0}, 0, \cdots, 0, b_{1}, b_{0}, 0, \cdots, 0\right),
$$

and furthermore G.C.D. $\left(a_{0}, b_{1}, b_{0}\right)=1$.

We now note the formulas

$\left(x_{1}, x_{2}, 0, \cdots, 0, y_{1}, y_{2}, 0, \cdots, 0\right) \mathfrak{T}_{S}=\left(x_{1}, x_{2}, 0, \cdots, 0, y_{1}+\lambda x_{1}, y_{2}, 0, \cdots, 0\right)$, where $S=\lambda \dot{+} 0^{(n-1)}$, and 
$\left(x_{1}, x_{2}, 0, \cdots, 0, y_{1}, y_{2}, 0, \cdots, 0\right) \Im_{J}=\left(-y_{1},-y_{2}, 0, \cdots, 0, x_{1}, x_{2}, 0, \cdots, 0\right)$,

where $J=0^{(n)}$. The alternate use of these formulas has the effect of setting up a Euclidean algorithm on the elements in the first and $(n+1)$ st positions. Therefore after a finite number of steps we have either

$$
\left(a_{1}, a_{2}, \cdots, a_{n}, b_{1}, \cdots, b_{n}\right) \sim(a, 0, \cdots, 0,0, c, 0, \cdots, 0)
$$

(where $c$ occurs in the $(n+2)$ nd position) or

$$
\left(a_{1}, a_{2}, \cdots, a_{n}, b_{1}, \cdots, b_{n}\right) \sim(a, b, 0, \cdots, 0,0,0, \cdots, 0),
$$

for some integers $a, b$, and $c$. In the former case observe that

$$
(a, 0, \cdots, 0,0, c, 0, \cdots, 0) \varsigma_{J}=(a,-c, 0, \cdots, 0,0,0, \cdots, 0),
$$

where $J=1+0^{(n-1)}$. In either case, therefore,

$$
\left(a_{1}, \cdots, a_{n}, b_{1}, \cdots, b_{n}\right) \sim(a, d, 0, \cdots, 0,0, \cdots, 0),
$$

where $a$ and $d$ are relatively prime. Now choose $V \in \Omega_{2}$ so that $(a, d) V=(1,0)$, and set $U=V+I^{(n-2)}$. Then

$$
(a, d, 0, \cdots, 0,0, \cdots, 0) \Re_{U}=(1,0, \cdots, 0,0, \cdots, 0) .
$$

This proves the result.

Theorem 1. Let $A_{1}$ and $B_{1}$ be $j \times n$ integral matrices, $j \leqq n$. Then $\left(\begin{array}{ll}A_{1} & B_{1}\end{array}\right)$ can be completed to an element of $\Gamma_{2 n}$ by placing $2 n-j$ rows below $\left(A_{1} B_{1}\right)$ if and only if $\left(A_{1}, B_{1}\right)$ is a normal pair.

Proof. If $\left(A_{1} B_{1}\right)$ is completable, trivially $\left(A_{1}, B_{1}\right)$ form a normal pair. We now prove the converse by induction on $n$. The result for $n=1$ is an immediate consequence of Lemma 2 ; let $n>1$, and assume that a normal pair of $t \times(n-1)$ integral matrices can be completed to an element of $\Gamma_{2(n-1)}$ for $t \leqq n-1$.

Since $\left(A_{1} B_{1}\right)$ is primitive, the G.C.D. of the elements of its first row is 1 . By Lemma 2 we have therefore

$$
\left(A_{1} B_{1}\right) \sim\left(\begin{array}{cccc}
1 & \mathfrak{n}^{\prime} & 0 & \mathfrak{n}^{\prime} \\
\mathfrak{x} & A_{2} & \mathfrak{y} & B_{2}
\end{array}\right)
$$

where $\mathfrak{x}$ and $\mathfrak{y}$ are $(j-1) \times 1$ vectors, and $\mathfrak{n}$ represents a null column vector whose size depends on the context. Since the right-hand side is a normal pair, the matrix

$$
\left(\begin{array}{ll}
1 & \mathfrak{n}^{\prime} \\
\mathfrak{x} & A_{2}
\end{array}\right)\left(\begin{array}{cc}
0 & \mathfrak{y}^{\prime} \\
\mathfrak{n} & B_{2}^{\prime}
\end{array}\right)=\left(\begin{array}{cc}
0 & \mathfrak{y}^{\prime} \\
\mathfrak{n} & \mathfrak{x} \mathfrak{y}^{\prime}+A_{2} B_{2}^{\prime}
\end{array}\right)
$$

must be symmetric; therefore $\mathfrak{y}=\mathfrak{n}$, and consequently $\left(A_{2}, B_{2}\right)$ form a normal 
pair of $(j-1) \times(n-1)$ matrices. By the induction hypothesis there exists a matrix

$$
\left(\begin{array}{ll}
R & S \\
T & U
\end{array}\right) \in \Gamma_{2(n-1)}
$$

with its first $(j-1)$ rows given by $\left(A_{2} B_{2}\right)$. Define

$$
\mathfrak{X}=\left(\begin{array}{ll}
1+R & 0+S \\
0+T & 1+U
\end{array}\right)
$$

Then $\mathfrak{X} \in \Gamma_{2 n}$, and

$$
\left(\begin{array}{cccc}
1 & \mathfrak{n}^{\prime} & 0 & \mathfrak{n}^{\prime} \\
\mathfrak{x} & A_{2} & \mathfrak{n} & B_{2}
\end{array}\right) \mathfrak{X}^{-1}=\left(\begin{array}{ccccc}
1 & \mathfrak{n}^{\prime} & & 0 & \mathfrak{n}^{\prime} \\
\mathfrak{x} & I^{(j-1)} & 0 & \mathfrak{n} & 0
\end{array}\right) .
$$

But the right-hand matrix consists of the first $j$ rows of $\Re_{V}$, where

$$
V=\left(\begin{array}{cccc}
1 & 0 & \cdots & 0 \\
\mathfrak{x} & I^{(j-1)} & & 0 \\
\mathfrak{n} & 0 & & I^{(n-j)}
\end{array}\right) .
$$

The theorem now follows by the use of Lemma 1.

THEOREM 2. The array given by (1) is completable if and only if it is normal.

Proof. As observed before, a completable array is obviously normal. Assume hereafter that the array given in (1) is normal, and (without loss of generality) that $j \geqq k$.

For, if the given array is a normal $(j, k)$ array with $j<k$, then

$$
\left(\begin{array}{cc}
C_{1} & D_{1} \\
-A_{1} & -B_{1}
\end{array}\right)
$$

is a normal $(k, j)$ array; if this latter array is completed to an element $Y \in \Gamma_{2 n}$, then the original array is completable to

$$
\left(\begin{array}{cc}
0 & -I^{(n)} \\
I^{(n)} & 0
\end{array}\right) \cdot Y \in \Gamma_{2 n}
$$

By Theorem 1 we have

$$
\left(\begin{array}{cc}
A_{1} & B_{1} \\
C_{1} & D_{1}
\end{array}\right) \sim\left(\begin{array}{ccc}
I^{(j)} & 0 & 0 \\
C_{2} & D_{2}
\end{array}\right)
$$

for some $C_{2}, D_{2}$. By Lemma 1 we see that $\left(C_{2}, D_{2}\right)$ form a normal pair, and furthermore

$$
\left(I^{(j)} 0\right) D_{2}^{\prime}=\left(I^{(k)} 0\right)^{\prime}
$$


so that we have

$$
D_{2}=\left(I^{(k)} 0^{(k, j-k)} X^{(k, n-j)}\right)
$$

for some $X$. Now set

$$
U=\left(\begin{array}{ccc}
I^{(k)} & 0 & 0 \\
0 & I^{(j-k)} & 0 \\
X^{\prime} & 0 & I^{(n-j)}
\end{array}\right),
$$

and observe that

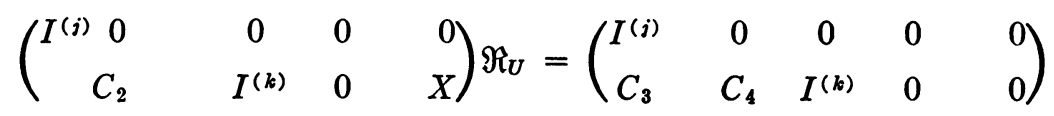

where $C_{3}$ is a $k \times j$ matrix, and $C_{4}$ a $k \times(n-j)$ matrix. Again using Lemma 1, the matrix

$$
\left(\begin{array}{llll}
C_{3} & C_{4}
\end{array}\right)\left(\begin{array}{lll}
I^{(k)} & 0 & 0
\end{array}\right)^{\prime}
$$

must be symmetric, so that

$$
C_{3}=\left(C_{31}^{(k, k)} C_{32}\right)
$$

with symmetric $C_{31}$. But now

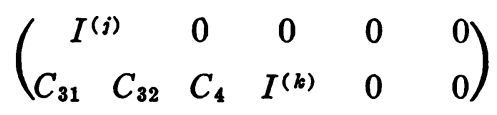

consists of the first $j$ rows and the $(n+1)$ st, $\cdots,(n+k)$ th rows of

$$
\left(\begin{array}{cccccc}
\multicolumn{2}{c}{I^{(j)}} & 0 & & 0 & \\
0 & I^{(n-j)} & & & \\
C_{31} & C_{32} & C_{4} & I^{(k)} & 0 & 0 \\
C_{32}^{\prime} & 0 & 0 & 0 & I^{(j-k)} & 0 \\
C_{4}^{\prime} & 0 & 0 & 0 & 0 & I^{(n-j)}
\end{array}\right) \in \Gamma_{2 n} .
$$

This proves the result.

2. We now turn to the problem of finding an expression for the general completion (5 of a given normal $(j, k)$ array, and we again assume without loss of generality that $j \geqq k$. If $\mathfrak{E}_{0}$ is a specific completion of the given array, then $\mathfrak{E}$ is a completion if and only if $\mathfrak{S} \mathcal{E}_{0}^{-1}$ is an element of $\Gamma_{2 n}$ whose first $j$ rows are given by

$$
\left(I^{(j)} 0^{(j, 2 n-j)}\right)
$$

and its $(n+1)$ st, $\cdots,(n+k)$ th rows by

$$
\left(0^{(k, n)} I^{(k)} 0^{(k, n-k)}\right) \text {. }
$$


Thus if $\mathfrak{X}$ represents the general such element of $\Gamma_{2 n}$, then $\mathfrak{X} \mathfrak{G}_{0}$ is the general completion of the given array.

Let us write

$$
\begin{aligned}
& \mathfrak{X}=\left(\begin{array}{llllll}
I & 0 & 0 & 0 & 0 & 0 \\
0 & I & 0 & 0 & 0 & 0 \\
E_{1} & E_{2} & E_{3} & F_{1} & F_{2} & F_{3} \\
0 & 0 & 0 & I & 0 & 0 \\
G_{4} & G_{5} & G_{6} & H_{4} & H_{5} & H_{6} \\
G_{1} & G_{2} & G_{3} & H_{1} & H_{2} & H_{3}
\end{array}\right) \begin{array}{l}
k \\
j-k \\
n-j
\end{array} \\
& \begin{array}{llllll}
k & j-k & n-j & k & j-k & n-j
\end{array}
\end{aligned}
$$

where we have indicated the numbers of rows and columns in the various submatrices. Then $\mathfrak{X} \in \Gamma_{2 n}$ if and only if $\mathfrak{X}$ is an integral matrix for which

$$
\left(\begin{array}{lll}
I & 0 & 0 \\
0 & I & 0 \\
E_{1} & E_{2} & E_{3}
\end{array}\right)\left(\begin{array}{lll}
0 & 0 & F_{1}^{\prime} \\
0 & 0 & F_{2}^{\prime} \\
0 & 0 & F_{3}^{\prime}
\end{array}\right) \text { and }\left(\begin{array}{lll}
0 & 0 & 0 \\
G_{4} & G_{6} & G_{6} \\
G_{1} & G_{2} & G_{3}
\end{array}\right)\left(\begin{array}{lll}
I & H_{4}^{\prime} & H_{1}^{\prime} \\
0 & H_{6}^{\prime} & H_{2}^{\prime} \\
0 & H_{6}^{\prime} & H_{3}^{\prime}
\end{array}\right)
$$

are symmetric, and

$$
\left(\begin{array}{lll}
I & 0 & 0 \\
0 & I & 0 \\
E_{1} & E_{2} & E_{3}
\end{array}\right)\left(\begin{array}{lll}
I & H_{4}^{\prime} & H_{1}^{\prime} \\
0 & H_{5}^{\prime} & H_{2}^{\prime} \\
0 & H_{6}^{\prime} & H_{3}^{\prime}
\end{array}\right)-\left(\begin{array}{lll}
0 & 0 & 0 \\
0 & 0 & 0 \\
F_{1} & F_{2} & F_{3}
\end{array}\right)\left(\begin{array}{lll}
0 & G_{4}^{\prime} & G_{1}^{\prime} \\
0 & G_{5}^{\prime} & G_{2}^{\prime} \\
0 & G_{6}^{\prime} & G_{3}^{\prime}
\end{array}\right)=I .
$$

These conditions give:

$$
\begin{aligned}
& F_{1}=0, F_{2}=0, E_{3} F_{3}^{\prime} \text { symmetric, } G_{4}=0, G_{1}=0, G_{5} H_{5}^{\prime}+G_{6} H_{6}^{\prime} \text { symmetric, } \\
& G_{2} H_{2}^{\prime}+G_{3} H_{3}^{\prime} \text { symmetric, } G_{5} H_{2}^{\prime}+G_{6} H_{3}^{\prime}=H_{5} G_{2}^{\prime}+H_{6} G_{3}^{\prime}, \\
& E_{1}=0, H_{4}=0, H_{1}=0, H_{5}=I, H_{2}=0, \\
& E_{2} H_{5}^{\prime}+E_{3} H_{6}^{\prime}-F_{3} G_{6}^{\prime}=0, E_{2} H_{2}^{\prime}+E_{3} H_{3}^{\prime}-F_{3} G_{3}^{\prime}=I .
\end{aligned}
$$

Hence we have

$$
\mathfrak{X}=\left(\begin{array}{llllll}
I & 0 & 0 & 0 & 0 & 0 \\
0 & I & 0 & 0 & 0 & 0 \\
0 & E_{2} & E_{3} & 0 & 0 & F_{3} \\
0 & 0 & 0 & I & 0 & 0 \\
0 & G_{5} & G_{6} & 0 & I & H_{6} \\
0 & G_{2} & G_{3} & 0 & 0 & H_{3}
\end{array}\right),
$$


with symmetric $E_{3} F_{3}^{\prime}, G_{5}+G_{6} H_{6}^{\prime}, G_{3} H_{3}^{\prime}$; where

$$
E_{2}=F_{3} G_{6}^{\prime}-E_{3} H_{6}^{\prime}, \quad G_{2}=H_{3} G_{6}^{\prime}-G_{3} H_{6}^{\prime}, \quad \text { and } \quad E_{5} H_{3}^{\prime}-F_{3} G_{3}^{\prime}=I .
$$

Therefore

$$
\left(\begin{array}{ll}
E_{3} & F_{3} \\
G_{3} & H_{3}
\end{array}\right) \in \Gamma_{2(n-j)}
$$

and so

$$
\left(\begin{array}{c}
E_{2} \\
G_{2}
\end{array}\right)=\left(\begin{array}{ll}
E_{3} & F_{3} \\
G_{3} & H_{3}
\end{array}\right)\left(\begin{array}{c}
-H_{6}^{\prime} \\
G_{6}^{\prime}
\end{array}\right)
$$

is true if and only if

$$
\left(\begin{array}{c}
-H_{6}^{\prime} \\
G_{6}^{\prime}
\end{array}\right)=\left(\begin{array}{rr}
H_{3}^{\prime} & -F_{3}^{\prime} \\
-G_{3}^{\prime} & E_{3}^{\prime}
\end{array}\right)\left(\begin{array}{l}
E_{2} \\
G_{2}
\end{array}\right) .
$$

We find easily that

$$
\begin{aligned}
G_{5}+G_{6} H_{6}^{\prime} & =G_{5}+\left(-E_{2}^{\prime} G_{3}+G_{2}^{\prime} E_{3}\right)\left(-H_{3}^{\prime} E_{2}+F_{3}^{\prime} G_{2}\right) \\
& =G_{5}+E_{2}^{\prime} G_{3} H_{3}^{\prime} E_{2}+G_{2}^{\prime} E_{3} F_{3}^{\prime} G_{2}-G_{2}^{\prime} E_{3} H_{3}^{\prime} E_{2}-E_{2}^{\prime} G_{3} F_{3}^{\prime} G_{2} .
\end{aligned}
$$

But $E_{3} H_{3}^{\prime}=I+F_{3} G_{3}^{\prime}$, so

$$
G_{5}+G_{6} H_{6}^{\prime}=G_{5}-G_{2}^{\prime} E_{2}+\text { symmetric matrix, }
$$

and therefore $G_{5}+G_{6} H_{6}^{\prime}$ is symmetric if and only if $G_{5}-G_{2}^{\prime} E_{2}=S$ is symmetric. We now observe that

$$
\mathfrak{X}=\left(\begin{array}{llll} 
& & & \\
& I & & 0 \\
0 & 0 & 0 & \\
0 & S & G_{2}^{\prime} & I \\
0 & G_{2} & 0 &
\end{array}\right)\left(\begin{array}{cccccc}
I & 0 & 0 & & & \\
0 & I & 0 & & 0 & \\
0 & E_{2} & I & & & \\
& & & I & 0 & 0 \\
& 0 & & 0 & I & -E_{2}^{\prime} \\
& & & 0 & 0 & I
\end{array}\right)\left(\begin{array}{ccc}
I+E_{3} & 0+F_{3} \\
& \\
0+G_{3} & I+H_{3}
\end{array}\right) .
$$

Hence, if $\mathfrak{E}_{0}$ is a specific completion of a given normal $(j, k)$ array, the general completion equals $\mathfrak{C}_{0}$ multiplied on the left by the above expression for $\mathfrak{X}$, where

$$
\left(\begin{array}{ll}
E_{3} & F_{3} \\
G_{3} & H_{3}
\end{array}\right)
$$

is an arbitrary element of $\Gamma_{2(n-j)}$, where $S$ is an arbitrary symmetric $(j-k)$ $\times(j-k)$ matrix, and where $G_{2}$ and $E_{2}$ are arbitrary $(n-j) \times(j-k)$ matrices. 
For the special case $j=n, k=0$ we obtain from the above Siegel's result that

$$
\mathfrak{C}=\left(\begin{array}{ll}
I & 0 \\
S & I
\end{array}\right) \mathfrak{S}_{0},
$$

with symmetric $S$.

We finally note that the above reasoning holds true for any Euclidean ring.

UNIVERSITY OF ILLINOIS,

URBANA, ILL. 